\title{
Comparison of the Efficacy of 3 Anti-Viral Regimens Containing Kaletra, Ribavirin and Sofosbuvir in COVID 19 Disease
}

\author{
Alireza Davoudi ${ }^{1}$, Narges Najafi ${ }^{1}$, Esmaeel Sphandyari ${ }^{1}$, Hamideh Abbaspour Kasgari ${ }^{2}$, Atefe Tayebi ${ }^{1}$, Azita Akbari ${ }^{1}$, \\ Mohammad Tabarestani ${ }^{1}$ and Amir Mohammad Beyzaee ${ }^{1, *}$
}

${ }^{1}$ Antimicrobial Resistance Research Center, Department of Infectious Diseases, Mazandaran University of Medical Sciences, Sari, IR Iran
${ }^{2}$ Department of Clinical Pharmacy, School of Pharmacy, Mazandaran University of Medical Sciences, Sari, Iran

"Corresponding author: Amir Mohammad Beyzaee, Ghaemshahr, Antimicrobial Resistance Research Center, Department of Infectious Diseases, Mazandaran University of Medical Sciences, Sari, Iran, Tel: 00989395362521; E-mail: amir_beyzaee@yahoo.com

Received: 07 Nov, 2020 | Accepted: 07 Dec, 2020 | Published: 14 Dec, 2020

Citation: Davoudi A, Najafi N, Sphandyari E, Kasgari HA, Tayebi A, et al. (2020) Comparison of the Efficacy of 3 Anti-Viral Regimens Containing Kaletra, Ribavirin and Sofosbuvir in COVID 19 Disease. Autoimmun Infec Dis 3(2): dx.doi.org/10.16966/2470-1025.129

Copyright: (C) 2020 Davoudi A, et al. This is an open-access article distributed under the terms of the Creative Commons Attribution License, which permits unrestricted use, distribution, and reproduction in any medium, provided the original author and source are credited.

\section{Abstract}

Background: New therapeutic options are urgently needed to handle the ongoing pandemic of coronavirus disease 2019 (COVID-19). As some antiviral therapeutic regimens are assumed to be helpful, we decided to compare the efficacy of kaletra, kaletra plus ribavirin, and kaletra plus ribavirin plus sofosbuvir, as three different therapeutic regimens in covid 19 patients.

Methods: This was an observational retrospective study in adults affected by COVID-19 admitted to the Ghaem Shahr Razi Hospital in Mazandaran Province, Iran. We examined medical records of 1033 patients with COVID 19, Between February 2 to March 20, 2020. According to the received antiviral regimens, patients were divided into three groups: 1) Kaletra, 2) Kaletra Plus Ribavirin, 3) Kaletra Plus Ribavirin Plus Sofosbuvir. They were compared in terms of the illness severity, the ICU admission, need for non-invasive ventilation and mechanical ventilation, and the mean duration of hospital stays and ICU stays.

Results: Based on the inclusion criteria, 571 cases were categorized into three groups: Kaletra $(n=471)$, Kaletra plus ribavirin $(n=78)$, and Kaletra plus ribavirin plus sofosbuvir $(n=22)$. The demographic factors were not significantly different between the groups $(P$-value $>00.05)$. There was significant difference between male and female frequencies $(56.40 \%$ vs. $43.60 \%$, respectively, P-value $=0.002) .62 .50 \%$ of patients aged 50 and older. Symptoms and signs were not significantly different among the three groups (P-value>0.05), except Sputum; Sputum was significantly more often found in patients of the Kaletra+Ribavirin+Sofosbuvir group ( $P$ value=0.039). According to the ICU admission, need for non-invasive ventilation and mechanical ventilation, and the mean duration of hospital stays and ICU stays, patients treated with kaletra plus ribavirin plus sofosbuvir regimen experienced highly better improvement than the other groups; although this difference was not statistically significant (P-value $>>0.05)$.

Conclusions: Kaletra plus ribavirin plus sofosbuvir could be considered as a promising anti-viral therapeutic regimen for COVID-19 patients.

Keywords: Anti-viral treatment; COVID 19; Kaletra; Ribavirin; Sofosbuvir

\section{Introduction}

In early December 2019, the first cases of pneumonia of unknown origin were reported in Wuhan, the capital of Hubei province of China which was caused by a new beta-corona virus [1]. The World Health Organization has named the disease COVID-19 [2]. Until now (23 May 2020) about 5103006 confirmed cases and 333401 deaths have been reported worldwide [3]. The clinical presentation of the disease has a wide spectrum which varies from asymptomatic or mild cases (in more than $80 \%$ ) to severe cases leading to acute respiratory syndrome and respiratory failure and death. In severe cases, the manifestations of the disease are related to the release of cytokines and the cytokine storm syndrome [4]. No drug has yet been reported to be safe and effective for the treatment of COVID- 19. However, a number of medications have been proposed as potential investigational therapies, many of which are now being or will soon be studied in clinical trials [5].There are many drugs, therapies tried and plasma therapy and mesenchymal therapy are amongst many [6,7].

An open-label study published in 2004, suggested that adding the lopinavir-ritonavir (kaletra) to ribavirin anti-viral regimen of patients with SARS significantly reduces the risk of adverse clinical outcomes (acute respiratory distress syndrome [ARDS] or death), as well as viral load in patients, in comparison with ribavirin alone [8,9]. These two drugs were recommended for moderate to severe illness in the first national protocol for the care of COVID19 in Iran. However, ribavirin was eliminated in later versions of this protocol [10].

Sofosbuvir could be considered as a potential option in the treatment of COVID-19 patients, based on the similarity between the replication mechanisms of the $\mathrm{HCV}$ and the coronavirus. 
Also, due to the lack of experimental evidences, it is hypothesized that sofosbuvir might be a potential option to improve care of patients with COVID-19 especially at the beginning of the disease and before invasion of the virus into the lung parenchymal cells [11].

As there is no evidence comparing the efficacy of kaletra, Ribavirin, and sofosbuvir, we aimed to evaluate the clinical outcomes of treating adult COVID 19 patients with kaletra, Ribavirin, and sofosbuvir, in this novel retrospective cohort study.

\section{Methods}

In an observational retrospective study, we examined medical records of 1033 patients with COVID 19 in terms of antiviral drugs use. These data were obtained from COVID 19 patients hospitalized in Qaemshahr Razi Hospital, the referral hospital for infectious diseases in the mazandaran province of iran, and the main university and educational center of the province for hospitalization of corona patients, from February 2 to March 20, 2020. The definitive diagnosis was based on a positive PCR test of the nasopharyngeal swab sample or the lung CT scan in accordance with COVID 19. Patients were divided into three therapeutic groups:1) Kaletra, 2) Kaletra Plus Ribavirin, 3) Kaletra plus Ribavirin plus Sofosbuvir, in which 90 to $95 \%$ of the cost of the medications mentioned above, is covered by insurances, with the help of Health system evolution plan of Health Ministry. The cases who were treated with any of our three study regimens were entered to the study. They were compared in terms of the Illness severity. Definitions of disease severity were based on Iranian national guidelines for the diagnosis and treatment of COVID 19 disease: 1- Mild stage 2- Moderate respiratory stage (patients whose arterial $\mathrm{O} 2$ saturation percentage is less than 94 and greater than or equal to 90) 3-Critical stage (cases with O2 Saturation less than or equal to $88 \%$, shock or multi organ failure, or patients needed noninvasive or mechanical ventilator respiratory support) [12].

\section{Measurements and statistical Analysis}

Analysis was performed using SPSS software (IBM SPSS Statistics for Windows, Version 22.0. Armonk, NY: IBM Corp). Quantitative variables were reported by mean and standard deviation (SD) and qualitative variables were reported using frequency and percentage. Because of the normal distribution of the data, the independent Oneway ANOVA was used to assess the means differences. Chi-square and Fisher exact tests were used to assess the statistical relationships between categorical variables. The level of significance was set as $\mathrm{P}$ value $<0.05$ for all analyses.

\section{Results}

\section{Demographic characteristics}

Based on the inclusion criteria, 571 cases were categorized into three groups: Kaletra $(n=471)$, Kaletra plus ribavirin $(n=78)$, and Kaletra plus ribavirin plus sofosbuvir $(\mathrm{n}=22)$. There was significant difference between male and female frequencies (56.40\% vs. $43.60 \%$, respectively, P-value $=0.002)$. The majority of patients $(93.00 \%)$ have a body mass index of less than $40 \mathrm{~kg}$ per $\mathrm{m} 2$ and $62.50 \%$ of patients aged 50 and older. The demographic factors such as age, gender, body mass index, past drug history, and smoking history were not significantly different between the three groups (P-value $>0.05)$ (Table 1). In the evaluation of past medical history, only chronic renal failure has a significantly higher rate in the group with Kaletra and ribavirin regimen $(\mathrm{P}$-value $=0.002)$.

\section{Clinical findings}

Table 2 illustrates clinical features of the patients in each group. Symptoms and signs were not significantly different among the three groups (P-value $>0.05$ ), except Sputum. Sputum was significantly more often found in patients of the Kaletra+Ribavirin+Sofosbuvir group ( $\mathrm{P}$ value $=0.039$ ).

\section{Treatment outcomes}

Table 2 shows treatment outcomes in each group. The ICU admission rate, need for non-invasive ventilation and mechanical ventilation were not significantly different among the groups (P-value $>0.05$, Chart 1). The mean duration of hospital stays and ICU stays did not show significant difference among the three groups ( $\mathrm{P}$-value $>0.05$ ).

\section{Discussion}

Up to now, no definitive therapeutic regimen is reported as a specific treatment for COVID-19, though some are under evaluation, including experimental antivirals.

Sofosbuvir, known as an anti-hepatitis C virus (HCV) drug, is able to suppress positive-strand RNA viruses; Flaviviridae and Togaviridae. Coronaviruses are one of the positive-strand RNA virus families with conserved polymerase. As a result, SARS-CoV-2 RdRp could be effectively inhibited by sofosbuvir [13].

Also, as sofosbuvir is a safe and well tolerated (at $400 \mathrm{mg}$ daily in a 24 week); and intracellular active metabolite of sofosbuvir is highly stable, it is hypothesized that SARS-CoV-2 infection could also be susceptible to sofosbuvir [13].

A study by Sadeghi A, et al. was performed to evaluate the efficasy of Sofosbuvir and Daclatasvir compared with standard care, in the treatment of moderate or severe coronavirus patients admitted to hospital, in a randomized controlled trial. 66 patients were entered to the study, either the treatment arm $(n=33)$ or the control arm $(n=33)$. The duration of hospital stay was significantly decreased in the treatment arm rather than the standard care alone. Also fewer deaths were reported in the treatment arm. Rate of hospital discharge in the treatment group was significantly higher than the control group (Gray's $\mathrm{P}=0.041$ ). Clinical recovery within 14 days was obtained by $29 / 33(88 \%)$ of the treatment arm and $22 / 33(67 \%)$ of the control arm $(\mathrm{P}=0.076)[14]$.

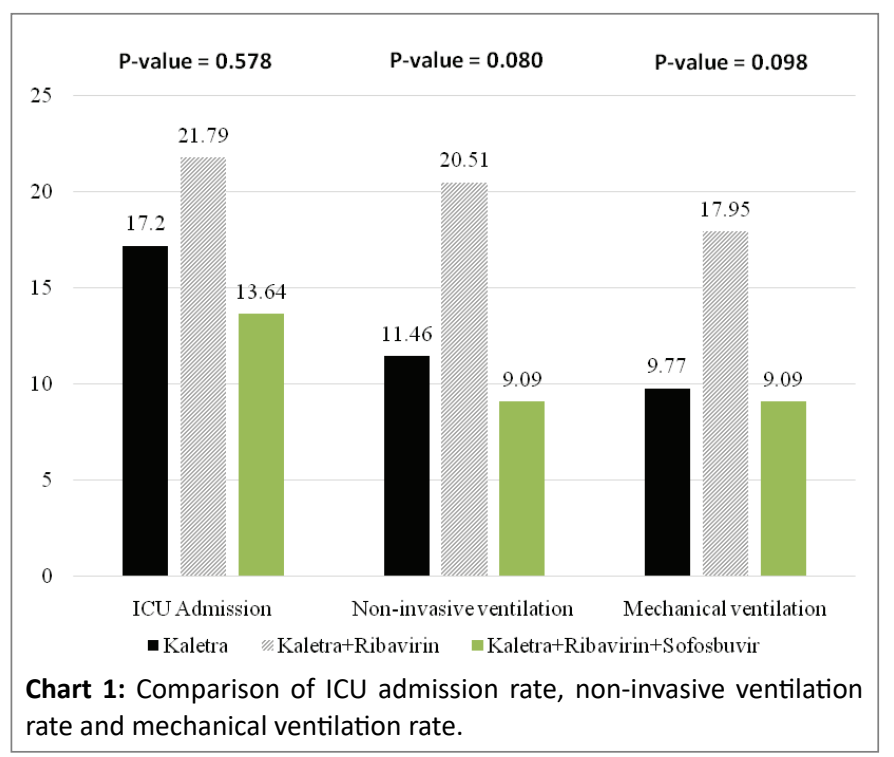


Table 1: Demographics, past medical history, Past drug history and smoking history.

\begin{tabular}{|c|c|c|c|c|c|c|}
\hline & & Kaletra & Kaletra+Ribavirin & Kaletra+Ribavirin+Sofosbuvir & & \\
\hline & & $(n=471)$ & $(n=78)$ & $(n=22)$ & Total & P-value \\
\hline \multirow{3}{*}{ Age, Year } & $<50$ & $177(37.58)$ & $28(35.9)$ & $9(40.91)$ & $214(37.48)$ & \multirow{3}{*}{0.874} \\
\hline & $50-65$ & 151(32.06) & $22(28.21)$ & $6(27.27)$ & 179(31.35) & \\
\hline & $>65$ & 143(30.36) & 28(35.9) & $7(31.82)$ & 178(31.17) & \\
\hline \multirow{2}{*}{ BMI (1), kg/m² } & $<40$ & 439(93.21) & 73(93.59) & 19(86.36) & 531(92.99) & \multirow{2}{*}{0.373} \\
\hline & $>40$ & $32(6.79)$ & $5(6.41)$ & $3(13.64)$ & $40(7.01)$ & \\
\hline \multirow{2}{*}{ Gender } & Female & $212(45.01)$ & $29(37.18)$ & $8(36.36)$ & $249(43.61)$ & \multirow{2}{*}{0.34} \\
\hline & Male & $259(54.99)$ & $49(62.82)$ & $14(63.64)$ & $322(56.39)$ & \\
\hline Smoker & & $10(2.12)$ & $0(0.00)$ & $1(4.54)$ & $11(1.93)$ & 0.208 \\
\hline \multirow{8}{*}{ PMH (2) } & Diabetes & $136(28.87)$ & $22(28.21)$ & $6(27.27)$ & 164(28.72) & 0.981 \\
\hline & Hypertension & $128(27.18)$ & $18(23.08)$ & $6(27.27)$ & $152(26.62)$ & 0.748 \\
\hline & CRF (3) & $11(2.34)$ & $8(10.26)$ & $2(9.09)$ & $21(3.68)$ & 0.002 \\
\hline & $\mathrm{CHF}(4)$ & $13(2.76)$ & $4(5.13)$ & $1(4.55)$ & $18(3.15)$ & 0.329 \\
\hline & IHD (5) & $68(14.44)$ & $8(10.26)$ & $0(0)$ & $76(13.31)$ & 0.091 \\
\hline & DLP (6) & $33(7.01)$ & $6(7.69)$ & $0(0)$ & $39(6.83)$ & 0.552 \\
\hline & Hypothyroidism & $29(6.16)$ & $7(8.97)$ & 2(9.09) & $38(6.65)$ & 0.437 \\
\hline & Other (7) & $127(26.96)$ & $24(30.77)$ & $3(13.64)$ & $154(26.97)$ & 0.284 \\
\hline \multirow{6}{*}{ PDH (8) } & Statins & $93(19.75)$ & $10(12.82)$ & $4(18.18)$ & 107(18.74) & 0.348 \\
\hline & Naproxen & $11(2.34)$ & $1(1.28)$ & $0(0)$ & $12(2.1)$ & 0.653 \\
\hline & Losartan & $72(15.29)$ & $11(14.1)$ & $5(22.73)$ & $88(15.41)$ & 0.603 \\
\hline & ACE inhibitor & $18(3.82)$ & $2(2.56)$ & $1(4.55)$ & $21(3.68)$ & 0.841 \\
\hline & Levothyroxine & $29(6.16)$ & $7(8.97)$ & 2(9.09) & $38(6.65)$ & 0.585 \\
\hline & Methadone & $10(2.12)$ & $3(3.85)$ & $0(0)$ & $13(2.28)$ & 0.49 \\
\hline
\end{tabular}

(1) Body Mass Index, (2) Past medical history, (3) Chronic renal failure, (4) Chronic heart failure, (5) Ischemic heart disease, (6) Dyslipidemia, (7) Others: Liver disease + Chemotherapy + Radiotherapy + Major thalassemia + Solid tumor + Asthma + Splenectomy + Multiple sclerosis + Corticosteroid therapy, (8) Past drug history

Also, direct access to antiviral drugs such as ribavirin that has an existing inventory and reliable supply chain, can be considered as a priority for treating patients infected by COVID-19. According to the in-vitro antiviral activity of ribavirin against SARS-CoV-2, and related strategies developed during the prior SARS and MERS outbreaks, ribavirin could strongly be considered as an agent to significantly impact on corona virus and slow the outbreak down [15].

On the other hand, a study by Wan $\mathrm{S}$, et al. was performed to observe the clinical features and treatment outcomes of COVID-19 patients in northeast Chongqing. They collected and analyzed epidemiological, clinical features, laboratory findings, radiological characteristics, treatment, and clinical outcomes of 135 COVID-19 patients. Patients were treated with different therapeutic regimens: All patients were treated with antiviral medications $(135$ [100\%]) (Kaletra and interferon were both used), antibacterial therapy (59 [43.7\%]), and corticosteroids (36 [26.7\%]). Also, most of the patients received traditional Chinese medicine (TCM) (124 [91.8\%]). They concluded that Kaletra and TCM played an important role in the treatment of the SARS-CoV-2 viral pneumonia. They suggested that patients should receive Kaletra early and should be treated by a combination of Western and Chinese medicines [16].

Combining antiviral agents has led to show a synergistic effect against COVID-19 virus in in vitro models [17]. Another study in China has reported satisfactory results from combination of ribavirin with lopinavir/ritonavir and interferon $\beta-1$ together [18].
A study by Kasgari HA, et al. was performed to evaluate the efficasy of sofosbuvir plus daclatasvir in combination with ribavirin for hospitalized COVID-19 patients with moderate disease compared with standard care; in a randomized controlled trial. 48 patients were recruited; 24 patients were randomly assigned to the intervention group and 24 to the control group. No patient in the intervention group died compared with three in the control group; however, these differences were not significant. Sofosbuvir/daclatasvir/ribavirin did not reduce the duration of hospitalization, but cumulative incidence of recovery was higher in the sofosbuvir/daclatasvir/ribavirin group compared with the control group. Fewer ICU admissions and deaths were observed in the sofosbuvir/daclatasvir/ribavirin arm; however, these differences were not significant [19].

Given the facts above, we decided to compare the efficacy of kaletra, kaletra plus ribavirin, and kaletra plus ribavirin plus sofosbuvir, as three different therapeutic regimens in COVID 19 patients, in an observational retrospective study.

We studied and analysed all the recorded dates of all 571 patients, including the ICU admission, need for non-invasive ventilation and mechanical ventilation, and the mean duration of hospital stays and ICU stays. According to the mentioned criteria's above, patients treated with kaletra plus ribavirin plus sofosbuvir regimen experienced highly better improvement than the other groups; although this difference was not statistically significant. 
Table 2: Symptoms, signs and outcomes.

\begin{tabular}{|c|c|c|c|c|c|c|}
\hline & \multirow{2}{*}{$\begin{array}{l}\text { Kaletra } \\
(n=471)\end{array}$} & \multirow{2}{*}{$\begin{array}{c}\text { Kaletra+Ribavirin } \\
(n=78)\end{array}$} & \multirow{2}{*}{$\begin{array}{c}\text { Kaletra+Ribavirin+Sofosbuvir } \\
\qquad(\mathrm{n}=22)\end{array}$} & \multirow[b]{2}{*}{ Total } & \multirow[b]{2}{*}{ P-value } \\
\hline & & & & & & \\
\hline \multirow{15}{*}{ Symptoms } & Fever, $\mathrm{n}(\%)$ & $94(60.65)$ & 29(69.05) & $3(50.00)$ & 126(62.07) & 0.779 \\
\hline & Chilling, $n(\%)$ & $58(37.91)$ & 19(46.34) & $2(33.33)$ & $79(39.5)$ & 0.649 \\
\hline & Dry cough, n(\%) & $77(50.33)$ & $19(46.34)$ & $3(50)$ & $99(49.5)$ & 0.304 \\
\hline & Sputum, n(\%) & $21(13.73)$ & $3(7.32)$ & $2(33.33)$ & $26(13)$ & 0.039 \\
\hline & Weakness, n(\%) & $42(27.45)$ & $19(46.34)$ & $0(0)$ & $61(30.5)$ & 0.349 \\
\hline & Sweating, n(\%) & $14(9.15)$ & $1(2.44)$ & $1(16.67)$ & $16(8)$ & 0.122 \\
\hline & Muscles pain, n(\%) & $43(28.1)$ & $17(41.46)$ & $1(16.67)$ & $61(30.5)$ & 0.668 \\
\hline & Shortness of breath, $\mathrm{n}(\%)$ & $14(9.15)$ & $7(17.07)$ & $1(16.67)$ & $22(11)$ & 0.437 \\
\hline & Dyspnea, n(\%) & $77(50.33)$ & $18(43.9)$ & $3(50)$ & $98(49)$ & 0.455 \\
\hline & Tachypnea, n(\%) & $13(8.5)$ & $2(4.88)$ & $2(33.33)$ & $17(8.5)$ & 0.619 \\
\hline & Throat ache, $\mathrm{n}(\%)$ & $5(3.27)$ & $0(0)$ & $0(0)$ & $5(2.5)$ & 1 \\
\hline & Gastrointestinal, n(\%) & $75(49.02)$ & $18(43.9)$ & $2(33.33)$ & $95(47.5)$ & 0.192 \\
\hline & Chest tightness and Chest pain, $\mathrm{n}(\%)$ & $7(4.58)$ & $5(12.2)$ & $0(0)$ & $12(6)$ & 0.237 \\
\hline & Headache and confusion, $n(\%)$ & $18(11.76)$ & $2(4.88)$ & $0(0)$ & $20(10)$ & 0.855 \\
\hline & Fatigue, $\mathrm{n}(\%)$ & $18(3.82)$ & $5(6.41)$ & $2(9.09)$ & $25(4.38)$ & 0.181 \\
\hline \multirow{5}{*}{ Signs } & Temperature, ${ }^{\circ} \mathrm{C}$ & $37.17(0.77)$ & $37.19(0.91)$ & $37.05(0.62)$ & $37.17(0.79)$ & 0.727 \\
\hline & $\mathrm{SBP}(1), \mathrm{mmHg}$ & $118.23(22.18)$ & $116.49(22.55)$ & $115(12.54)$ & $117.87(21.91)$ & 0.672 \\
\hline & $\mathrm{DBP}(2), \mathrm{mmHg}$ & $72.46(13.31)$ & $71.42(12.21)$ & $71.82(10.53)$ & $72.29(13.05)$ & 0.806 \\
\hline & RR (3), count/minute & $20.56(5.31)$ & $21.56(9.6)$ & $19.9(2.61)$ & $20.68(6.03)$ & 0.35 \\
\hline & Heart rate, Beat/minute & $92.41(21.55)$ & $93.96(18.47)$ & $92.32(29.46)$ & $92.62(21.48)$ & 0.84 \\
\hline \multirow{3}{*}{$\begin{array}{c}\mathrm{O}_{2} \\
\text { Saturation, } \\
\mathrm{n}(\%)\end{array}$} & $>93$ & $290(61.57 \%)$ & $45(57.69 \%)$ & $15(68.18 \%)$ & $350(61.3 \%)$ & \multirow{3}{*}{0.854} \\
\hline & $90-93$ & $106(22.51 \%)$ & $21(26.92 \%)$ & $5(22.73 \%)$ & $132(23.12 \%)$ & \\
\hline & $<90$ & $75(15.92 \%)$ & $12(15.38 \%)$ & $2(9.09 \%)$ & $89(15.59 \%)$ & \\
\hline \multirow{5}{*}{ Outcomes } & ICU (4) Admission, $\mathrm{n}(\%)$ & $81(17.2 \%)$ & $17(21.79 \%)$ & $3(13.64 \%)$ & $101(17.69 \%)$ & 0.578 \\
\hline & Non-invasive ventilation (6), $\mathrm{n}(\%)$ & $54(11.46 \%)$ & $16(20.51 \%)$ & $2(9.09 \%)$ & $72(12.61 \%)$ & 0.08 \\
\hline & Mechanical ventilation, $\mathrm{n}(\%)$ & $46(9.77 \%)$ & $14(17.95 \%)$ & $2(9.09 \%)$ & $62(10.86 \%)$ & 0.098 \\
\hline & Hospital stay (days), M(SD) (5) & $3.81(3.22)$ & $5.04(4)$ & $4.75(4.35)$ & $4.75(4.26)$ & 0.566 \\
\hline & ICU length of stay (days), M(SD) (5) & $6(3.61)$ & $5.27(5.85)$ & $6.14(4.99)$ & $5.99(5.06)$ & 0.806 \\
\hline
\end{tabular}

(1) Systolic blood pressure, (2) Diastolic blood pressure, (3) Respiratory rate, (4) Intensive care unit, (5) Mean (Standard deviation). (6) Nasal $\mathrm{O}_{2}$ or Mask $\mathrm{O}_{2}$ or CPAP or BIPAP

However, there are limitations to our study, most importantly, the number of patients in each group was different and it is hard to identify probable beneficial effects on survival.

Lack of blinding is one of the other limitations of our study. While we found clinical improvement benefits, we were not able to analyse neither biological markers of improvement as we did not measure viral decay nor serological inflammatory markers over time, which would both be helpful data to demonstrate effective antiviral therapy.

\section{Conclusion}

Kaletra plus ribavirin plus sofosbuvir could be considered as a promising anti-viral therapeutic regimen for COVID-19 patients.

\section{References}

1. Guan W, Ni Z, Hu Y, Liang WH, Ou CQ, et al. (2020) Clinical Characteristics of Coronavirus Disease 2019 in China. N Engl J Med 382: $1708-1720$.

2. World Health Organization (2020) Rolling updates on Coronavirus disease (COVID-19).
3. World Health Organization (2020) Coronavirus disease (COVID-19) Pandemic. Situation reports 124 .

4. Taisheng Li (2020) Diagnosis and clinical management of severe acute respiratory syndrome Coronavirus 2 (SARS-CoV-2) infection: an operational recommendation of Peking Union Medical College Hospital (V2.0). Emerg Microbes Infect 9: 582-585.

5. World Health Organization (2020) Off-label use of medicines for COVID-19. Scientific brief.

6. Sahu KK, Siddiqui AD, Cerny J (2020) Mesenchymal Stem Cells in COVID-19: A Journey from Bench to Bedside. Lab Med.

7. Sahu KK, Jindal V, Siddiqui AD, Cerny J, Gerber JM (2020) Convalescent Plasma Therapy: A Passive Therapy for an Aggressive COVID-19. J Med Virol.

8. Chu CM, Cheng VCC, Hung IFN, Wong MML, Chan KH, et al. (2004) Role of lopinavir/ritonavir in the treatment of SARS: initial virological and clinical findings. Thorax 59: 252-256.

9. $\quad$ Cao B, Wang Y, Wen D, Liu W, Wang J, et al. (2020) A Trial of LopinavirRitonavir in Adults Hospitalized with Severe COVID-19. N Engl J Med 382: $1787-1799$. 
10. Azadmanesh K, Jamaati H, Javanian M (2020) Iranian national guidelines for the diagnosis and treatment of COVID 19 disease at the level of outpatient and inpatient services.

11. Nourian A, Khalili $H(2020)$ Sofosbuvir as a potential option for the treatment of COVID-19. Acta Biomed 91: 236-238.

12. Azadmanesh K, Jamaati $H$, Javanian M (2020) Iranian national guidelines for the diagnosis and treatment of COVID 19 disease at the level of outpatient and inpatient services. $7^{\text {th }}$ Edition.

13. Sayad B, Sobhani M, Khodarahmi R (2020) Sofosbuvir as repurposed antiviral drug against COVID-19: why were we convinced to evaluate the drug in a registered/approved clinical trial? Arch Med Res 51: 577-581.

14. Sadeghi A, Asgari AA, Norouzi A, Kheiri Z, Anushirvani A, et al. (2020) Sofosbuvir and daclatasvir compared with standard of care in the treatment of patients admitted to hospital with moderate or severe coronavirus infection (COVID-19): a randomized controlled trial. J Antimicrob Chemother 75: 3379-3385.

15. Khalili JS, Zhu H, Mak NSA, Yan Y, Zhu Y (2020) Novel coronavirus treatment with ribavirin: Groundwork for an evaluation concerning COVID-19. J Med Virol 92: 740-746.
16. Wan S, Xiang Y, Fang W, Zheng Y, Li B, et al. (2020) Clinical features and treatment of COVID-19 patients in northeast Chongqing. J Med Virol 92: 797-806.

17. Lo HS, Hui KP, Lai HM, Khan KS, Kaur S, et al. (2020) Simeprevir suppresses SARS-CoV-2 replication and synergizes with remdesivir. BioRxiv.

18. Hung IF-N, Lung K-C, Tso EY-K, Liu R, Chung TW-H, et al. (2020) Triple combination of interferon beta- $1 \mathrm{~b}$, lopinavir-ritonavir, and ribavirin in the treatment of patients admitted to hospital with COVID-19: an open-label, randomised, phase 2 trial. Lancet 395: 1695-1704.

19. Kasgari HA, Moradi S, Shabani AM, Babamahmoodi F, Badabi ARD, et al. (2020) Evaluation of the efficacy of sofosbuvir plus daclatasvir in combination with ribavirin for hospitalized COVID-19 patients with moderate disease compared with standard care: a singlecentre, randomized controlled trial. J Antimicrob Chemother 75: 3373-3378. 